water vapour, which of course would be sufficient to account for that large effect.

Sir William Crookes mentions that, according to his experiments, water vapour produces a greater conduction at very low pressures than air; but I decidedly disbelieve the difference to be in any way similar to the numbers given by $\mathrm{Mr}$. Brush, in consequence of a reasoning mentioned at the end of my Phil. Mag. paper, and I think the simplest explanation to be afforded by this source of errors vitiating the pressure indications.

It is no mere hypothesis but a certain fact that water vapour is being evolved by heated glass, and probably many other substances. Sir William Crookes gathers quite a number of arguments for it from his own researches, amongst others spectroscopical proofs. The same opinion was put forward by Kundt and Warburg, who were led to it by the very same sort of experiments on conduction of heat as Mr. Brush's, which they made as early as 1875 (Poggendorff's Annalen, 156, p. 177). Further investigations on the hygroscopical properties of glass, and on means for partially removing them, were published by Warburg and Ihmori (Wiedemann's Annalen, 27, p. 48I).

I cannot prove, of course, that there is no new gas evolved, but I maintain that whatever facts Mr. Brush has put forward as an evidence for its existence, can be explained quite simply by the presence of water vapour (perhaps also other condensable vapours), which he seems to have overlooked. I do not think it necessary to go into details, and to analyse more thoroughly the -rather fantastic - speculative part of the paper, where scarcely any statement is not open to serious objections.

Although thus I differ from $\mathrm{Mr}$. Brush very much in respect to the interpretation of his recent results, I think his elaborate experimental investigations, as reported in his Phil. Mag. paper, to be of great value for the theory of these phenomena; certainly it would be very desirable that he might carry on those researches, as he promised there to do.

Vienna, University.

$$
\text { M. Smoluchowski de Smolan. }
$$

\section{The Curve of Life.}

THE relationship between the duration of adolescence and potential longevity in different species of mammals has repeatedly been the subject of speculation. M. P. Flourens, in his work

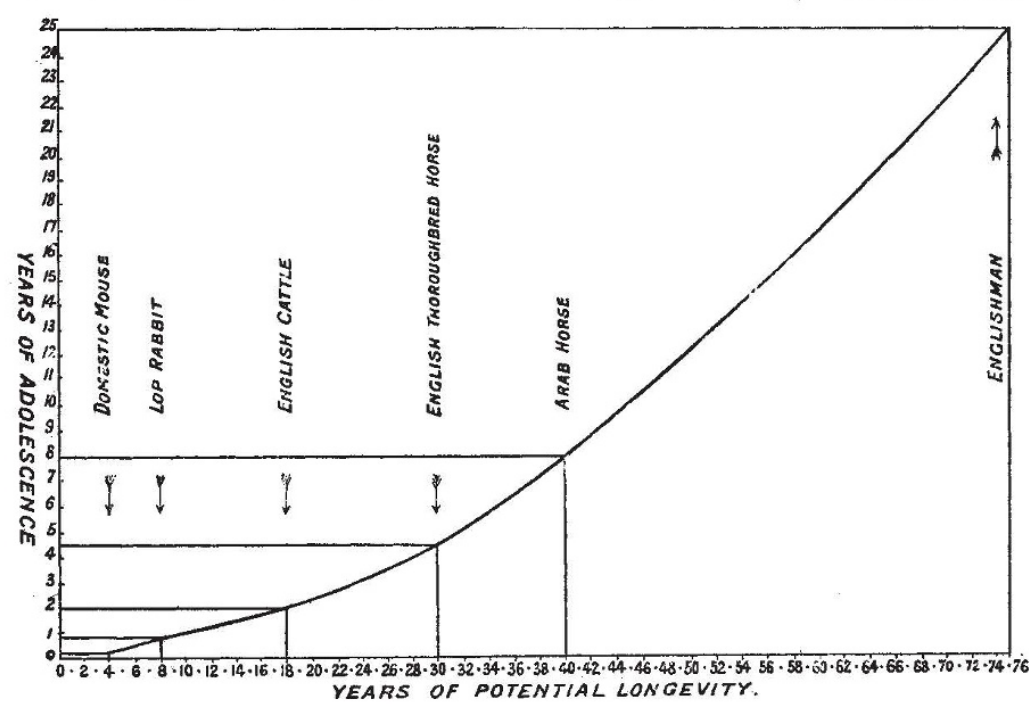

on "Human Longevity," made the ratio between the two periods as I to 5 ; Buffon had previously concluded that it was as 1 to 7 . In neither case were the data sufficiently numerous and trustworthy to make these figures generally accepted. In the course of some investigations on the variability of the adolescent period in different breeds of the same species among certain well-known mammals, I have satisfied myself that a relationship exists between the duration of growth and the length of an animal's natural life ; although it is evidently not of the kind suspected by the older writers. It may be stated as follows:-The ratio of length of adolescence to length of life in the NO. I 523 , VOL. 59] shortest lived mammals is proportionally less than it is in longer lived mammals. For example, the period of growth and development of the domestic mouse is, according to my informant, a breeder of these small rodents, about three months. Its natural lifetime is four years. In other words, the mouse may be expected to live about fifteen times its adolescent period as a mature animal. The Arab horse, according to a well-known authority, arrives at maturity in about eight years, its lifetime is about forty years; that is to say, the animal lives four times the length of its adolescence as an adult. Man, on the other hand, who only completes his growth by the union of the sternal epiphysis of the clavicle to its shaft at the age of twenty-five, has, after passing his fiftieth year, or "the middle arch of life," to use Dr. Farr's phrase, only another twenty-five years' expectation of life. His potential longevity accordingly foreshadows a period of maturity not greater than twice the length of his youth.

I have obtained, through the kindness of numerous correspondents interested in breeding and rearing of farm and other domestic animals, the approximate lengths of these two periods in a few well-known mammals; and the accompanying diagram shows the relations between growth and longevity among the same animals plotted as a definite curve. This result was entirely unexpected by me, and it may be interesting to some of your readers.

W. Ainsile Hollis.

Hove.

The Alleged Destruction of Swallows and Martins in Italy.

IN your issue of December 22, I898, I read the report of a conference held by the Society for the Protection of Birds, at which a paper was read on the decrease of swallows and martins coming to England, giving, as a reason for this decrease, the netting of thousands of these birds on their arrival at the Italian shores, and their subsequent consumption as food.

May I venture to remark that, during a residence of some years in Italy, I have never once seen a swallow, or any member of its family, exposed for sale, and that $I$ have never known, or heard of, an instance of their being netted in the manner described, though I am well acquainted with nearly every part of the Peninsula.

Since reading the above mentioned accusation against Italy, I have asked several Italians whether they knew of such a practice, and am informed that it is simply non-existent, the swallow being, perhaps, the one bird in this country which is regarded with a kind of sentiment by all classes, as the harbinger of spring.

Swallows, moreover, do not arrive on these coasts in a state of exhaustion, and to net them would be no easy feat.

A few isolated cases of the cruel method of capturing them with artificial flies may occur, but not more so than in England.

The Italian may be ruthless in his destruction of other birds, but is certainly not a destroyer of the Hirundinidae.

Of the similar charge made against the French, I am not in a position to judge, but I imagine that the cause for the decrease of the Hirundinidae in England may lie in quite another direction, and may be attributable to some equivalent decrease of their favourite insects in our islands, or in some atmospheric and climatic change. Italy, I am convinced, is not responsible in any way for it. RICHARD BAGOT. Roma, December 27, 1898.

\section{RADIATION PHENOMENA IN THE MAGNETIC FIELD.}

$\mathrm{N}$ the spring of 1897 the scientific world became indebted to Dr. Zeeman for the observation that when a source of light is placed in a strong magnetic field the spectral lines of the light emitted by that source suffer 
marked modification. The general type, or characteristic type, of this modification is that when the slit of the spectroscope views the sources of light across the lines of magnetic force, each spectral line becomes a triplet, of which the middle line has the same wave-length as the original line; whereas the side lines of the triplet have wave-lengths, respectively, a little longer and a little shorter than that of the unmodified line, the difference of wave-length being proportional to the strength of the magnetic field. Further, the central line has its vibrations parallel to the lines of force, whereas the side lines of the triplet have their vibrations perpendicular to the lines of force. Thus, if the axis of the magnetic field is horizontal, so that the lines of force are horizontal, and if the slit of the spectroscope looks horizontally across the lines of force, then in the central constituent of the triplet the vibrations are horizontal, while in the side lines the vibrations are vertical. Thus the central line is plane polarised, and the side lines are also plane polarised, but in a perpendicular plane. This is the typical phenomenon when the light is viewed across the lines of force. When the light is viewed along the lines of force - that is, through axial holes pierced in the pole-pieces of the electromagnet, the modification is different. In this case, instead of a triplet with plane polarised constituents, we are presented with a doublet, having circularly polarised constituents. That is, each spectral line is broken up into two lines of slightly different wave-length; one constituent being circularly polarised in one sense, and the other in the opposite sense. As before, the difference of wave-length, and therefore the separation of the constituents of these doublets in the spectroscope, is proportional to the strength of the magnetic field for each line, but differs in amount for the different spectral lines.

In order to fix the ideas of those who are not familiar with this department of physics, the phenomena described above are represented diagrammatically in Fig. $I$. Thus

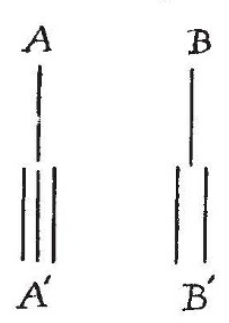
at $\mathrm{A}$ the upper single line is supposed B to represent a bright spectral line of some substance when the radiating source is not influenced by the magnetic field. This line becomes converted into three distinct lines, that is a triplet, as shown underneath at $\mathrm{A}^{\prime}$, when the source of light is subject to a strong magnetic field, and the radiation takes place across the lines of force. If $\mathrm{N}$ be the vibration frequency FIs. I. of $\mathrm{A}$, then the vibration frequencies of
the members of the triplet $\mathrm{A}^{\prime}$, into
$\mathrm{A}$ is converted, are $\mathrm{N}-n, \mathrm{~N}, \mathrm{~N}+n$, where $\mathrm{N}$ FIs. I. of A, then the vibration frequencies of
the members of the triplet $\mathrm{A}^{\prime}$, into
A is converted, are $\mathrm{N}-n, \mathrm{~N}, \mathrm{~N}+n$, where $\mathrm{N}$ which $\mathrm{A}$ is converted, are $\mathrm{N}-n, \mathrm{~N}, \mathrm{~N}+n$, where $\mathrm{N}$
is a small quantity depending on the strength of the magnetic field. On the other hand, when the source of light is viewed along the lines of force a bright spectral line, $\mathrm{B}$, becomes converted into a doublet, $\mathrm{B}^{\prime}$, consisting of two distinct lines which are circularly polarised in opposite senses. The constituents of the triplet $\mathrm{A}^{\prime}$ are, on the contrary, plane polarised, the direction of vibration in the middle line being horizontal, while that in the side lines is vertical.

The foregoing are the phenomena demanded by the simplest form of theory, and they are the phenomena actually yielded by experiment in the case of the vast majority of spectral lines. Many lines, however, when carefully examined in a sufficiently strong magnetic field, yield phenomena which differ in a remarkable manner from the simple theoretical expectation described above. In some cases the middle line of the triplet becomes resolved into a pair of lines so that the triplet becomes a quartet, while in other cases each line of the triplet becomes a pair, and thus a sextet is produced; and in some cases the side lines of the triplet become resolved into triplets, while the middle line becomes a doublet, and then an octet is produced, and so on. Thus generally, when the light is viewed across the lines of force, we may say a single spectral line becomes resolved by the magnetic field into a system of lines consisting of a central part bordered by two side parts. The central part may consist of one or more lines, and is plane polarised, while the side parts may each consist of one or more lines, and are also plane polarised in a plane at right angles to the plane of polarisation of the central part.

On account of this opposite polarisation the central part may be quenched and the sides examined separately, or vice versa, by means of a nicol's prism, and consequently the existence of this plane polarisation enables us to scrutinise the phenomena much more closely and effectively than would be otherwise possible unless, indeed, a magnetic field of any desired strength could be produced so as to obtain complete and wide separation of the various constituents of the modified line. But it is not possible at present to produce a magnetic field for working purposes of a strength exceeding 30,000 to 40,000 C.G.S. units. Hence the polarisation is of importance for purposes of nbservation. The best way to take advantage of it is not to use a nicol's prism (which lets through only one of the two plane polarised beams), but to use instead a double image prism, or a rhomb of doubly refracting crystal, placed before the slit of the spectroscrope, so that two images of the source are produced on the slit, one above the other (the slit being supposed vertical). Of these images one consists of light vibrating horizontally - that is, it consists of the light which forms the central part of the triplet $A^{\prime}$ (Fig. I), while the other image consists of light vibrating vertically- that is, the light which forms the sides of the triplet $\mathrm{A}^{\prime}$ when the magnetic field is excited. These two images on the slit give rise to two spectra in the field of view of the spectroscope, one above the other: one consisting of the lines which form the centres of the triplets, and the other of the lines which form the sides. This is shown in Fis. 2, where A represents a triplet as seen in the field of view, without the use of any nicol or double image prism, and $\mathrm{A}^{\prime}$ represents what is seen when a double image prism is used. The upper line in $A^{\prime}$ represents the light vibrating horizontally, and is what would, be seen if a nicol's prism were placed before the slit with its principal plane vertical; whereas the two lines below in $\mathrm{A}^{\prime}$ are formed by the light vibrating

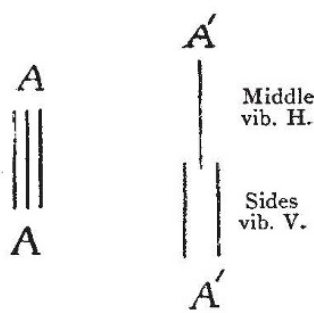

Fig. 2. vertically, and constitute what would be seen were the nicol turned through a right angle. With the double image prism, however, the upper and the lower lines. in $\mathrm{A}^{\prime}$ are seen simultaneously, and so a great deal of trouble is avoided, and much time is saved when the phenomena are being photographed. But the chief advantage of separating the middle from the side lines, as at $\mathrm{A}^{\prime}$ (Fig. 2), lies in the fact that in many cases the difference of wave-length of the middle and the side lines is so small, even in a very strong magnetic field, that the width of the lines causes them to overlap, and so obliterate the phenomena. It was for this reason that in the earlier experiments made by Dr. Zeeman, merely a broadening of the spectral lines was observed, and not a tripling. In fact, it was not until theory pointed out that tripllng and plane polarisation should exist across the lines of force, that Zeeman interposed a nicol's prism, and found that the broadened line exhibited the polarisation required, and that the facts were not discordant with the theory. It is to be observed, however, as I have pointed out elsewhere, that the removal of the central part from the broadened line by a nicol properly interposed (so that 
the broadened line now appears as a doublet), does not absolutely prove that the broadened line is a triplet with its components overlapped. It merely determines that the broadened line may be a triplet, and that the theory which anticipates the tripling may be correct. In order to place this matter beyond all doubt, it is necessary to so increase the strength of the magnetic field that the components of the triplet (if they exist) shall be completely separated from one another; and when this ${ }^{1}$ is done, it is found that the tripling exists, but it is also found that many divergencies from the uniform expectation of theory (pure tripling) exist. Thus, as pointed out above, many lines under the influence of the magnetic field show as quartets, or sextets, or octets, or other modified form of the normal triplets. In the examination of these cases the double image prism forms a very valuable adjunct, as all the light polarised in one plane goes to form one image, while all the light polarised in the perpendicular plane forms the other image. The appearance presented in the field of view of the spectroscope by different types of lines, under these circumstances, is shown in Fig. 3. In this figure the lines of the upper row are formed by one image from the double image prism-that is to say, by the light vibrating horizontally, and correspond to the central members of the normal triplets; while the bottom row consists of light vibrating vertically, and represents the side lines of the normal triplets. Thus at $A A^{\prime}$ we have the normal triplet, as expected by theory, with the central line, $A$, polarised in one plane, while the

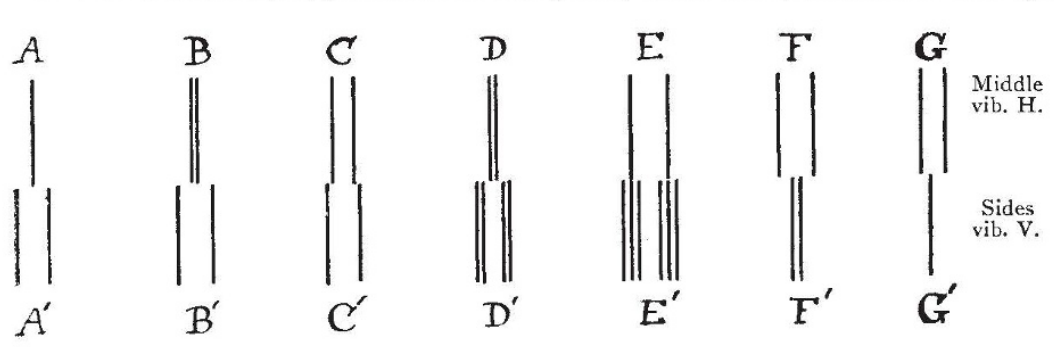

FIG. 3.

two side lines, $\mathrm{A}^{\prime}$, are polarised in the perpendicular plane. This type exists in the case of by far the greater number of spectral lines, and may be regarded as the general or normal type, if for no other reason than the frequency with which it occurs. The second type, shown at B B', is a quartet in which, instead of a single middle line, we have two middle lines close together at $B$, with the two side lines at $\mathbf{B}^{\prime}$ as before. This type of quartet occurs in the blue cadmium line 4800 , and in the blue zinc line 4722. At $\mathrm{C} \mathrm{C}^{\prime}$ another species of quartet is shown; in this there are two middle lines also, but the separation of these is almost as wide as that of the side lines, so that the appearance presented to the eye when the double image prism is not used is that of two fine doublets, rather than the quartet appearance of the type $\mathrm{B} \mathrm{B}^{\prime}$. This third type, $\mathrm{CC}^{\prime}$, occurs in the case of the sodium line $D_{1}$, the greenish-blue line of barium 4934, and many others. The fourth type, $D D^{\prime}$, is a sextet of fine uniformly spaced lines, two of which correspond to each component of the normal triplet. That is, the central component is a doublet, and each of the side components is also a doublet. This type is represented by the line $\mathrm{D}_{2}$ of sodium. The fifth type is shown at $\mathrm{EE}^{\prime}$, where the central constituent is a doublet, and each of

1 This was effected by the writer in October 1897 , and triplets and wuartets were then photographed directly without the aid of a nicol or any polarising apparatus whatever. (See letter to NATURE, dated November I9, 1897 , vol. lvii. p. I73.) These photographs were shown at the November meeting of the Dublin University Experimental Science Association, at the December I897 meeting of the Royal Dublin Society, and at the January 1898 meeting of the Royal Society of London; but it was not until April 1898 that they were reproduced in the Philosophical Magazine (5th series, vol. xlv. p. 325 , plate xxiii.).

$$
\text { NO. I } 523 \text {, VOL. 59] }
$$

the side components is a triplet. The distance between the components of the central doublet in this case is about the same as that between the central members of the side triplets. This type is represented in the yellow line of barium 5850. All the variations so far noted may be embraced in the general statement that each line of the normal triplet $\mathrm{A} \mathrm{A}^{\prime}$ may itself become a doublet or a triplet.

The question now of greatest importance is whether these various types of modification by the magnetic field are consistent with the theoretical explanations of the phenomena put forward by Larmor, Lorentz, and others? Naturally one must endeavour to reconcile facts and theory. If this reconciliation has not yet been effected, we must not hastily conclude that the theory is wrong, or even that it requires to be modified or patched up ; and it was with this feeling that I put forward (Phil. Mag., ser. 5, vol. xlv. p. 325 , April 1898 ) the idea that these various modifications might be due to reversalthat is, to absorption in the outer parts of the spark or other source of light. Thus B (Fig. 3) might arise from A by reversal of the middle line, and so also might $\mathrm{CC}^{\prime}$ and $\mathrm{DD}^{\prime}$ be produced, and even $\mathrm{EE}^{\prime}$ might be intelligible from this hypothesis if we supposed double reversal (?) to occur in the side components of the triplet $\mathrm{AA}^{\prime}$, and a wide absorption band to occur in the middle line (supposed much broader than the others). But (as I stated when putting forward this view) the appearance presented to the eye is not that of ordinary reversal, so that appearances are against the supposition that the modifications are due to absorption in the vapour surrounding the source of light. But still it is to be remembered that the magnetic field exerts a considerable influence on the source of light, and might alter considerably the appearance of an ordinary reversal. However, in order to test this matter, I observed many lines, which de viate from the normal triplet type, in a magnetic field of gradually increasing strength. The object of this was to determine if the separation of the lines form. ing the upper row in Fig. 3 (say, the doublet $\mathrm{B}$ or $\mathrm{C}$ ) depended on the strength of the magnetic field. Thus, if the components of the doublet $\mathrm{B}$ remain fixed while the distance between the side lines $\mathrm{B}^{\prime}$ continues to increase as the magnetic field increases in strength, then we might conclude that reversal is not only a possible explanation by very probably the true explanation. But the components of the central parts $B, C, D$, do not remain fixed as the magnetic field increases in strength. On the contrary, the distance between the two lines $B$ increases as the strength of the field increases; indeed, as far as rough observations go, the distance between the components of $\mathrm{B}$ or $\mathrm{C}$, like the distance between the side lines $\mathrm{B}^{\prime}$ or $\mathrm{C}^{\prime}$, is proportional to the strength of the magnetic field. Similar remarks apply to the types DD', $\mathrm{EE}^{\prime}$, \& $\mathrm{C}$. When the field increases in strength, the lines forming D separate from each other, and so also do the doublets $\mathrm{D}^{\prime}$, and the lines forming each component of the latter also separate, so that the sextet remains a system of equally spaced lines. On the other hand, when the field is reduced in strength the various lines close up till B, C, D, E each appears as a single line with $\mathbf{B}^{\prime}, \mathrm{C}^{\prime}, \mathrm{D}^{\prime}$ as narrow doublets-in fact, the normal triplet type is approached in appearance as the field is reduced.

It appears, therefore, that the explanation of the various modifications of the normal triplet type cannot be satisfactorily explained by reversal, and consequently these divergencies must be referred to the action of the magnetic field on the vibrating structure which emits the 
radiation. Now the theory which indicates that a spectral line should be slit up into a pure triplet by the action of the magnetic field, assumes that the freedom of vibration is the same in all directions, and it is from this that the resolution into triplets occurs. This assumption is that which one most naturally makes in a first attack on a problem of this nature, but no one making it would be surprised if the facts did not turn out more complicated than the prediction of such a solution. For example, it is quite possible to conceive a state of affairs in which the magnetic field may constrain all vibrations to take place along the lines of force, in which case the side lines of the triplet would vanish; or, on the other hand, vibration in the direction of the lines of force might be impossible, in which case the central line of the triplet would vanish. Indeed, one is somewhat surprised that deviations of this kind from the normal triplet type do not more frequently occur. In fact, when I first examined the spectrum of iron, I hoped to find many deviations of this kind, but failed to detect any very marked difference between the behaviour of iron and other substances. This is not much to be wondered at when it is remembered that iron ceases to be magnetic at a comparatively low temperature, and, therefore, at the temperature of the spark of an induction coil, one should not expect its vapour to behave much differently from that of any other substance.

However, as already stated, the normal triplet type arises in theory because the orbit of the vibrating electron is supposed free from constraints and perturbations-that is, that movement is equally free in all directions. When constraints are imposed, or new forces arise which cause perturbations in the orbit, new frequencies will be introduced into the vibrating system. Thus if an electron, or an atom, or a particle describes an ellipse under a central force with frequency $\mathrm{N}$, and if disturbing forces came into play which cause the apse line to rotate with frequency $n$, then, as Dr. Stoney ${ }^{1}$ has shown, a spectral line arising from the original vibration of frequency $\mathrm{N}$ will become replaced by two others of frequencies $\mathrm{N}+n$ and $\mathrm{N}-n$ respectively. Again, if the disturbing forces cause a precessional motion of the plane of the orbit round a fixed line with frequency $n$, the original vibration of frequency $\mathrm{N}$ becomes replaced by three others of frequencies $\mathrm{N}+n, \mathrm{~N}$, and $\mathrm{N}-n$ respectively, and similar phenomena arise when other periodic disturbances occur in the orbital motion. We are prepared, therefore, to find that each line of the normal triplet may become itself a doublet or a triplet. ${ }^{2}$

The disturbing forces arising from the action of the magnetic field should increase with the strength of the field, so that if the distance between components of the doublet $B$ or C or D or E (Fig. 3), which takes the place of the central line of the normal triplet, should increase with the magnetic field, as it is found to do by experiment. In fact, if the distance between the side lines of the normal triplet $\mathrm{AA}^{\prime}$ be written in the form $d_{1}=k_{1} \mathrm{H}$, where $\mathrm{H}$ is the strength of the field, and $k_{1}$ a quantity depending on the wave-length and other constants involved in the production of the particular line in question, then the distance between the components of the modified central component B, C, \&c., may be written in the form $d_{2}=k_{2} \mathrm{H}$. Thus as the field increases in strength the whole system of lines into which any given spectral line becomes resolved, separate laterally from each other proportionately, as it were, according to a given scale Similar remarks, of course, apply to systems like $\mathrm{DD}^{\prime}$ and $\mathbf{E E}^{\prime}$.

Now in any particular case, such as $\mathrm{BB}^{\prime}$ for example, if the distance between the pair of lines $B^{\prime}$ is

1 Dr. G. J. Stoney, Trans. Roy. Dub. Soc., Vol. iv Series 2, p. 563 $189 \mathrm{~T}$. This is a very important paper when considered in connection with the above-mentioned magnetic perturbations of the spectral lines. 2 These matters are treated in further detail in a paper by the present
writer to appear in the forthcoming number of the Philosophical Magazine. while the distance between the

whe distance between the pair $\mathrm{B}$ is

$$
d_{2}=k_{2} \mathrm{H}
$$

there is apparently no reason why $k_{1}$ should be greater than, or less than $k_{2}$. Whether $k_{1}$ is greater than or less than $k_{2}$, must be determined by the action of the magnetic field on the system which produces the particular spectral line in question. Accordingly we are prepared to find that in some lines the components of the central line, as at $\mathrm{B}$, shall be much closer together than the side components at $\mathrm{B}^{\prime}$, while in others, as at $\mathrm{C}, \mathrm{E}$ and $\mathrm{F}$, the distance $d$., is nearly equal to, or may be even greater than the distance $d_{1}$ between the side lines. Thus, once the production of a quartet of the type $\mathrm{BB}^{\prime}$ is explained, all the other modifications become intelligible. The case in which the components $\mathrm{F}$ are wider apart than the side lines $\mathrm{F}$ (so that the centre, as it were, encloses the sides) is merely the same phenomenon (only more accentuated) as that shown at $\mathrm{BB}^{\prime}$ where the separation $d_{2}$ is less than $d_{1}$ This point is mentioned here specially because in some cases the separation $d_{2}$ is actually greater than $d_{1}$, and it seems to be regarded as a difficulty of a much higher order than that in which occurs in the ordinary quartet, where $d_{2}$ is less than $d_{1}$.

Lines of the former type $\mathrm{FF}^{\prime}$, viz. that in which $d$., is greater than $d_{1}$, seem to have been first observed by MM. Henri Becquerel and H. Deslandres (see Comptes rendus, t. 126, p. 997, April 4, I898) in the spectrum of iron, and subsequently Messrs. J. S. Ames, R. F. Earhart and $\mathrm{H}$. M. Reese announced that they had observed the form $\mathrm{GG}^{\prime}$ (Fig. 3) in the spectrum of iron. In this type the side lines $\mathrm{G}^{\prime}$ coincide, or are not sensibly separated. while the components of the central part G are well separated. ${ }^{1}$ (see Astro. Phys. Journat, vol. viii. p. 48 June 1898 ). The form in which this observation was described was calculated to startle, if not confound, the most firm believer in theory. It was said that these lines exhibited reversed polarisation-that is, that the polarisation of the centre is that which should occur in the sides, and vice versa. Stated in this way it is rather calculated to take one's breath away, but when stated as in the foregoing, it loses all special significance, viz. that it is merely a case of $d_{2}$, being greater than $d_{1}$, that is $k_{2}>k_{1}$, or a quartet in which the distance between the horizontally vibrating constituents is greater than the distance between the vertically vibrating constituents. Stated in this way it falls into line with the other phenomena, and is reduced to the explanation of the doubling of any one individual member of the normal triplet.

Other similar modifications have been observed by MM. Becquerel and Deslandres, who appear to have examined the spectrum of iron very thoroughly as well as the bands of carbon and cyanogen. These bands they found to be unaffected by a magnetic field strong enough to sensibly split up the air lines.

Investigations demanding special attention are those of Prof. A. A. Michelson, both on account of his reputation as an original investigator and by reason of the nature of the apparatus which he employed. Working with his interferometer, Prof. Michelson concluded some years ago (Phil. Mag., vol. xxxiv. p. 280, I892) that the spectral lines themselves instead of being, as ordinarily supposed, narrow bands of approximately uniform illumination from edge to edge, are on the contrary in most cases really complexes, some of them being ciose triplets, and so on. This structure has never yet been observed by means of any ordinary form of spectroscope, and accordingly it has been suggested that it does not

1 I have not yet observed this type, nor do my photographs verify the conclusion of Messrs. Ames, Earhart and Reese, regarding the lines mentioned by them as belonging to this type. (This is further referred to in the forthcoming number of the Phil. Mag.)

NO. I 523 , VOL. 59$]$ 
exist in the light radiated from the source, but is imposed on the spectral lines.by the apparatus used, namely, the interferometer. Be this as it may, the application of this instrument to the study of radiation phenomena in the magnetic field is highly interesting. In his first experiments Michelson merely observed a doubling of the spectral lines both along and at right angles to the lines of force (!), but subsequent observations proved that tripling occurred across the field of force, and that the constituents of the triplets were themselves multiple lines (see Astro. Phys. Journal, vol. vi. p. 48, 1897; vol. vii. p. I31, I898; vol. viii. p. 43, I 898). But this is accompanied by the most surprising statement that the separation of the lines in the triplets produced by the magnetic field is independent of both the spectral line and the substance. In other words, that the separation is the same for all lines and all substances! Now, in all observations with ordinary grating or prism spectroscopes the separation of the components produced by the magnetic field varies very considerably for the different spectral lines of the same or of different substances. Even in the case of lines of nearly the same wave-length the difference is often very marked. The separation not only differs for different substances, but it is some complex function of the wave-length for any one substance. That the interferometer has led to such a law as that announced by Prof. Michelson, shows that there is some peculiarity of the instrument not yet taken into account-or else that by chance Prof. Michelson has happened to confine his observations to lines which give approximately the same separation; yet this latter could not be easily done. Be this as it may, Michelson has examined these phenomena by aid of another new instrument of his own design--the Echelon spectroscope (Astro. Phys. Journal, vol. viii. p. 43, I898). With this instrument he states that the results previously obtained by aid of the interferometer, and the visibility curve, were confirmed. And this is striking, for if it confirms the general law stated by him in regard to the separation of the components, then the interferometer and the Echelon spectroscope are at variance with all other forms of spectroscope.

With apparatus which reveals structure or multiplicity in the ordinary spectral lines, it is to be expected that multiplicity would be readily revealed in the constituents produced by the magnetic field; yet in the case of some lines, the amount of finer structure revealed does not appear to be as great as that observed with a good grating, and this with other discrepancies require clearing up. If we suppose that an ordinary spectral line really consists of two or more very close lines, not separated in ordinary spectroscopes, and if we suppose that this multiplicity is produced by small perturbations caused by events inside the molecule, then it is clear that the further perturbations (if any) brought about by the magnetic field, may either increase, or diminish, or possibly reverse, those previously existing in the free field. And from this point of view the following most interesting observations made by Michelson (loc. cit.) become intelligible. "A very remarkable effect is observed in the case of the yellow copper line. This line without the field is a close double, the distance being $1 / 15$ oth of the distance between the $D$ lines, or $0.04 \mathrm{~A} . \mathrm{V}$. As the field increases the lines merge together without broadening, and with a strong field there is but a single narrow line."

"The behaviour of the yellow-green line of manganese is even more striking. The line is a quadruple line, just resolvable. In a weak magnetic field the light accumulates in the centre of the group, the lines becoming indistinct and merging together. In a strong field the quadruple band is reduced to a single fine line at the centre of the group."

In conclusion, it is necessary to mention briefly some ingenious methods which have been devised to exhibit the existence of the Zeeman phenomena in comparatively weak magnetic fields. The first of these chronologically was devised by M. Cotton (Comptes rendus, t. I25, p. 865) in 1897 , and depends upon the fact that if a small sodium flame, A, be placed in front of a larger one, B, and viewed against it, the outer edges of the small flame appear dark. This arises, as is well known, from the absorption which takes place in the outer sheath of the smaller flame. If, however, the flame $B$ be placed in the magnetic field, the dark border around A disappears. This arises from the fact that the magnetic field induces new periods of vibration in B (the side lines) which are not possessed by $\mathrm{A}$, and therefore not absorbed.

The next experiment to be mentioned is one of special elegance, devised by Prof. Auguste Righi (Comptes rendus, t. 127, p 2 16, 1898, and Rend. della R. Accad. dei Lincei, July i898). If a plane polarised beam of light from a powerful source, such as an arc lamp, be transmitted through an absorbing vapour, such as a sodium flame, or sodium vapour in a tube, and if the light, after passing through the vapour, be transmitted through a nicol's prism, and then received on the slit of a spectroscope, a continuous spectrum will be observed in which dark lines occur corresponding to the absorption lines or bands of the vapour. If the analysing nicol be rotated till its principal plane is perpendicular to that of the polariser, then all light in the spectroscope will be extinguished. 1 Now suppose this to be so arranged, and suppose, further, that the absorbing vapour is between the pole-pieces of a magnet so as to be subject to the action of the magnetic field, and suppose that the light passes through this vapour along the lines of magnetic force by passing through axial holes pierced in the polepieces, then under these circumstances, if the magnet be excited, bright lines appear in the spectroscope corresponding to the absorption lines of the vapour. At first sight it appears as if the magnetic field caused the vapour to emit its own vibrations as if it were highly luminous. It is not so, however. The explanation is that the magnetic field so affects the vapour, that if it were selfluminous any spectral line appertaining to it of frequency $\mathrm{N}$ is converted into two other vibrations of frequency $\mathrm{N}+n$ and $\mathrm{N}-n$ respectively; and these two, along the lines of force, are circularly polarised in opposite senses, and consequently the vapour when cold possesses the power of absorbing vibration of frequencies $\mathrm{N}+n$ and $\mathrm{N}-n$. Now the beam from the electric arc passing through the vapour being continuous, possesses vibrations of frequency $\mathrm{N}+n$ and also of frequency $\mathrm{N}-n$. These vibrations in the arrangement, described above, are plane polarised, and any plane polarised vibration is equivalent to two opposite circular vibrations. The result is that the vapour absorbs one of the circular components from the rectilinear vibration $\mathrm{N}+n$, and transmits the other. In the same way it also absorbs one of the circular component vibrations from the vibration $\mathrm{N}-n$, and transmits the other. These transmitted circular components are very intense (having evidently half the intensity possessed by the arc light), and they cannot be extinguished by the analysing nicol, so they consequently appear in the spectroscope. If the magnetic field is not very strong, the vibrations $\mathrm{N}+n$ and $\mathrm{N}-n$ practically coincide with $\mathrm{N}$, and what is presented to the eye is that the absorption lines of the vapour become bright when the magnetic field is excited. This can be observed in fields of very small intensity.

Prof. Righi mentions that the phenomenon observed in the foregoing experiment does not occur when the light traverses the vapour in a direction perpendicular to the lines of force. This is a result which differs from

1 If a sodium flame be used as the absorber, then of course faint sodium lines will still remain. For this reason the sodium flame used should not be bright. 
the theoretical expectation. For an emission frequency $\mathrm{N}$ of the vapour will now be converted, across the lines of force, into absorption frequencies $\mathrm{N}+n, \mathrm{~N}$, and $\mathrm{N}-n$. The first and last being for vertical vibrations, and the central one for horizontal vibrations. If, therefore, the plane of polarisation of the incident light (arc lamp) be inclined at any angle $a$ to the vertical, its horizontal component will be absorbed by the vapour for the frequency $\mathrm{N}$, and its vertical component for the frequencies $\mathrm{N}+n$ and $\mathrm{N}-n$. The other components will be transmitted, and being vertical and horizontal respectively, and not being of the same period, they cannot be extinguished by a nicol set to quench light polarised at an angle $a$ to the vertical. When the in cident light is polarised in a vertical plane, however, or in a horizontal plane, the analysing nicol can quench the transmitted light, and the lines do not light up in the spectroscope. The writer has found on trial that the expectation of theory is realised, and that when the polariser is inclined to the vertical the phenomenon takes place across the lines of force as in Righi's experiment along the lines of force. ${ }^{1}$

Many other interesting points deserve notice, such as Prof. G. F. Fitzgerald's theory connecting the Faraday effect with the Zeeman effect; but want of space compels us to close the present account of the work done in this field during the past year. We may just mention, in conclusion, that the Faraday effect in gases has been placed in strong evidence by an interesting experiment due to MM. Macaluso and Corbino (Comptes rendus, t. I27, p. 548, 1898), which depends for its explanation on the fact that the rotatory power of a substance increases enormously as the frequency of the transmitted light approaches that of an absorption band of the substance through which it is transmitted.

Thomas Preston.

\section{RECENT WORK IN COMPARATIVE MYOLOGY.}

$T \mathrm{HE}$ introduction of biology into medical education, productive of such good effects, has been in few departments more beneficial than in that of comparative anatomy; and we desire to call attention to the present aspect of one outcome of this, which appears to us a direct result of the adoption by the surgical anatomist of the comparative method, and to be full of promise for the future.

Our remarks are prompted by a paper which has recently appeared in the Proceedings of the Zoological Society, as the completion of an extensive study of the comparative myology of the terrestrial carnivora, by Prof. Bertram Windle and Mr. F. G. Parsons, a most laborious piece of work, involving the careful dissection of close upon one hundred individuals representative of the leading carnivorous families. Of desultory descriptions of the myology of individual mammals we have long had enough, and it is the merit of the two anatomists named to have attacked the subject in a thoroughly systematic manner, transcending that of most of their predecessors. Incorporating with their own extensive observations the sum of our previous knowledge, by carefully classifying the muscles, dealing with them in sets, and tabulating their relationships where necessary in a manner permitting of ready reference, they have now laid the foundation of a really comprehensive system of recording myological facts. The paper to which we refer is serial with others which its authors have published on the Rodentia and Marsupialia, either individually or together; and perusal of the series leads us to believe

1 Prof. Righi's elegant experiment was brought before the notice of the British Association in September last by Prof. S. P. Thompson, and three or four days afterwards, with kind permission, I made the observations here described in Prof. Barrett's laboratory in the Royal College of Science,

NO. I 523 , VOL. 59] that in finally deciding the zoological position of some of the more anomalous mammalian forms the study of the muscles may yet play a not unimportant part. We welcome thus, for example, the conclusion of one of the authors that the Jerboas are allied to the Myomorpha as insisted upon by Winge; and the fact that in other respects they are in agreement with bis recent work in the classification of the Mammalia, the value and importance of which has been by no means sufficiently appreciated in this country.

In having access to the collections of the Zoological Society, Royal College of Surgeons Museum, and other London institutions, our authors command a wealth of material unobtainable elsewhere, and they are thus enabled not merely to record facts of adult anatomy, but, by comparing individuals of species, to approximately determine the limitations of individual variation. Accurate observations, when systematically arranged, have a permanent value in the progress of science; and, confessing to an admiration of the laborious persistence with which our authors have persevered in their task, we sincerely hope they will continue it until each and every order of Mammals shall have been investigated.

\section{REV. BARTHOLOMEW PRICE, F.R.S.}

THE death of the Rev. Bartholomew Price, F.R.S., on Thursday last, deprives the University of Oxford of one who worked long and loyally for its welfare. $\mathrm{He}$ took a most active part in the business of the University and of his College, and on many occasions helped to further the interests of science at Oxford. For these labours and for his attractive personal qualities he will be long remembered by many old pupils and friends.

Dr. Price was born in 1818 at Coln St. Dennis, Gloucestershire, and was educated privately at Pembroke College, whence he obtained a first class in mathematics in 1840 . He gained the University Mathematical Scholarship in 1842 , and two years later was elected Fellow of his Coliege. In 1844 he became tutor, and nine years afterwards Sedleian Professor of Natural Philosophy. In 1852 appeared the first volume of his elaborate work on the infinitesimal calculus, dealing with the differential calculus; the second, on the integral calculus and calculus of variations, was published in I854; the third, on statics and dynamics of a particle, appeared in 1856 ; and the last of the four, on the dynamics of material systems, was published in 1862 . This treatise obtained for him a considerable reputation in the mathematical world; but his principal work in life was practical, and he will be remembered rather as the active secretary of the University Press during the years of its first great activities after the death of Dean Gaisford, than as a Mathematical Professor. Prof. Price resigned the secretaryship of the Clarendon Press in 1885 , when he was succeeded by Mr. P. Lyttelton Gell, who held the post till a few months ago.

Dr. Price was appointed Sedleian professor of natural philosophy at Oxford in 1853 , and he only retired from his post in June last, upon attaining his eightieth year. The event was commemorated by a dinner, at which numerous old pupils and others showed the esteem in which they held their counsellor and friend. For many years, both before and after his appointment to the chair of Natural Philosophy, the greater part of the mathematical teaching of the University was in his hands. He was one of the public examiners in mathematical and physical sciences eleven times in twenty-four years, and his works on the differential and integral calculus, \&c., were for long the recognised text-books.

Dr. Price was elected a Fellow of the Royal Society in I 852 , and he served on the Council of the Society no less than five times. He also served on the Royal Com- 